\title{
DEL TEATRO ÁUREO A LA ZARZUELA DECIMONÓNICA: ENTRE BOBOS ANDA EL JUEGO Y DON LUCAS DEL CIGARRAL ${ }^{1}$
}

\author{
Gerardo Fernández San Emeterio \\ Universidad Complutense de Madrid \\ gerarfer@ucm.es
}

\section{RESUMEN}

Don Lucas del Cigarral es una zarzuela de Tomás Luceño y Carlos Fernández Shaw, basada en Entre bobos anda el juego, de Rojas Zorrilla. Son escasas las adaptaciones de títulos de la Comedia áurea a la Zarzuela en el XIX y éste es uno de los pocos que tuvo éxito. Este artículo estudia la adaptación que sufrió el texto de Rojas para convertirlo en zarzuela.

\section{AbStRact}

Don Lucas del Cigarral is a «zarzuela» by Tomás Luceño and Carlos Fernández Shaw, based in the «comedia» Entre bobos anda el juego by Rojas Zorrilla. Recasting of «comedias» from Spanish Golden Age to the XIX Century «zarzuela» was not frequent and Don Lucas was one of the cases with success. This paper studies the way in which Rojas' text was recasted in order to make a «zarzuela» from it.

${ }^{1}$ Este artículo forma parte del proyecto Ópera, drama lírico y zarzuela grande entre 1860 y 1936. Textos y música en la creación del teatro lírico nacional, Referencia HAR2012-39820-c03-02 del Ministerio de Economia y Competitividad. Dirección General de investigación y gestión del Plan Nacional de I+D+i. Subproyecto dirigido por M. ${ }^{a}$ Pilar Espín Templado. 
Palabras Clave: Zarzuela, adaptación, Francisco de Rojas Zorrilla, Tomás Luceño, Carlos Fernández Shaw.

No son muy frecuentes los casos en los que la zarzuela decimonónica se inspiró directamente en el teatro del Siglo de Oro. Son más frecuentes los acercamientos a los siglos XVI y XVII a partir del trabajo de poetas románticos: el caso de Margarita, la tornera, de Carlos Fernández Shaw y Chapí, a partir de la leyenda homónima de Zorrilla, es el más conocido ${ }^{2}$.

El primer motivo de esta falta de adaptaciones de la dramaturgia clásica al teatro lírico, en mi opinión, se encuentra en las convenciones de ambos géneros, demasiado arraigadas en el público para que pareciera natural su intercambio. De forma similar a lo que sucedió con el Don Juan Tenorio de Zorrilla, donde la crítica vio en su adaptación lírica (con música de Nicolás Manent sobre texto del propio Zorrilla) «una profanación del drama original, [...] un robo a saco [...] de una obra tan arraigada en el alma del pueblo» ${ }^{3}$, cabe pensar si nuestros dramaturgos prefirieron dejar de lado la «brava mina» de Moreto y, aunque en su versificación fueran abundantes los estilemas de la Comedia, apartarse de una tradición que había supuesto un profundo debate estético e ideológico en las primeras décadas del siglo ${ }^{4}$, para acercarse a la fórmula de opéra-comique francesa, que dio frutos como Jugar con fuego, El juramento, Catalina, Pan y toros, El barberillo de Lavapiés o El rey que rabió, por citar sólo algunos casos.

Pensemos que ni siquiera Barbieri, tan buen conocedor de la literatura de los Siglos de Oro, realizó en su dilatada carrera como compositor un acercamiento directo a alguna comedia del XVII 5 .

Con todo, no faltaron casos aislados en los que poetas y compositores volvieron los ojos a la dramaturgia del XVII para atraerla al formato, ya establecido en los años finales del XIX, de la zarzuela grande, si bien rara se vieron coronados por el éxito de público ${ }^{6}$. M. ${ }^{a}$ Pilar Espín considera que «la presencia de los clásicos en la zarzuela la podríamos resumir en tres tipos de obras: [...] las refundiciones y adaptaciones más o menos libres; [...] la pervivencia y/o paralelismo de ciertos elementos y ambientación de obras clásicas en zarzuelas originales», así como a través de parodias. En el mismo artículo, la profesora Espín se ha referido a ellos considerando que «en la mayoría de los casos»su

\footnotetext{
${ }^{2}$ Creo, a pesar de ello, que es más habitual la conexión en nuestro teatro lírico que en el inglés, francés o alemán.

3 A. Peña, 1986, p. 26, n. Esta opinión, como señala Ma Pilar Espín Templado (1993, p. 305) «se desprende desde el momento de su estreno (crítica de la Ilustración Española y Americana, 8, noviembre, 1877), hasta la opinión de la crítica moderna (Alonso Cortés, 1943)»: el drama de Zorrilla «había arraigado [...] tan profundamente en el público, desde un primer momento, que cualquier variación de sus versos, o de sus personajes, no iba a ser fácilmente tolerada por los espectadores treinta y tres años después» (1993, p. 305). Para la bibliografía que este título ha suscitado, véase el mismo trabajo, p. 304, n. 14.

4 Me he referido a este aspecto en G. Fernández San Emeterio, 2012, pp. 117-121.

${ }^{5}$ Recordemos que, simultáneamente, tal y como afirma M. ${ }^{a}$ Pilar Espín Templado, «en el afán por dar con un espectáculo nacional, genuinamente español, no dudaron en volver al acervo de nuestra tradición líricodramática [...]. En efecto, ahí estaban unas piezas populares, unos bailes, unas canciones que los autores [...] recogieron ávidamente, plasmándolas en obras que se caracterizaron por su breve extensión -un acto-, sus personajes populares y una intención cómica.» (Espín Templado, 1987, p. 26).

${ }^{6}$ Dejo de lado deliberadamente el caso de Arrieta, al poner en música el texto de un auto de Calderón dentro de los actos del centenario de la muerte del dramaturgo, toda vez que se trata de un encargo oficial que no iba a competir en la cartelera con el resto de la producción lírica del momento.
} 
intención era la de «ennoblecer el teatro lírico mediante un libreto de calidad literaria y prestigio dramático» ${ }^{7}$. Se trata de un intento similar al que intentaría unos años más tarde Gregorio Martínez Sierra con su «teatro de arte».

\section{De la Comedia a la Zarzuela}

De entre esos títulos, me voy a ocupar en esta ocasión de Don Lucas del Cigarral, refundición de Entre bobos anda el juego preparada por Tomás Luceño y Carlos Fernández Shaw, con música de Amadeo Vives. Se estrenó en el Circo Parish el 19 de enero de 1899 y formaba parte de una temporada que Cotarelo calificó como «próspera» y en la que, tras la reposición de Marina, se estrenaron, entre otras obras, María del Carmen, de Feliú y Codina y Granados, y Curro Vargas, con texto de Joaquín Dicenta y Manuel Paso (a partir de El niño de la bola, de Pedro Antonio de Alarcón) y música de Ruperto Chapí. El propio Cotarelo reconoce que se trató de «una campaña muy fructífera para el cultivo del género grande, mientras los demás teatros madrileños se desvivían por el género chico» ${ }^{8}$.

\section{Pervivencia en la escena de la comedia de Rojas}

Acierta la profesora Menéndez Onrubia cuando plantea la posibilidad de que un texto teatral pase «al canon literario» si ha tenido «un buen texto escénico» (creo que debemos entenderlo como «adaptación» o «refundición») y un actor que encarnase un determinado personaje con la suficiente maestría como para influir en sus sucesivas representaciones» ${ }^{9}$. Al menos, con Entre bobos anda el juego, estas condiciones se cumplen.

De la producción de Rojas, según la misma autora, «en la década de 1854-1855 sólo cuatro de sus obras se vieron en los escenarios madrileños». Se trata de Donde hay agravios, no hay celos (en la refundición de Hartzenbuch titulada El amo criado), Lo que son mujeres, refundida por Gorostiza, Del rey abajo, ninguno y, finalmente, Entre bobos anda el juego ${ }^{10}$. De hecho, desde 1878, Entre bobos no había desaparecido del cartel del Teatro Español de Madrid ${ }^{11}$.

Esta permanencia se debía, sin duda, a la presencia del actor Donato Jiménez, que había hecho suyo el personaje, tal y como ha estudiado Carmen Menéndez Onrubia.

\footnotetext{
${ }^{7}$ M. ${ }^{a}$ Pilar Espín Templado, 2009, p. 1096.

8 E. Cotarelo, Ensayo, pp. 876-877.

${ }_{9}$ C. Menéndez Onrubia, 2007a, p. 406.

${ }^{10}$ C. Menéndez Onrubia, 2007b, p. 224.

${ }_{11}$ Pueden verse los datos recopilados por C. Menéndez Onrubia, 2007b, p. 406-407, n.
} 


\section{Don LUCAS DEL CIGARRAL ${ }^{\text {I2 }}$}

Como ya he señalado, la elección del título para convertirlo en zarzuela tuvo que deberse a que la comedia se había mantenido en el repertorio y ello permitía al público conocer la trama y apreciar los cambios que en ella introdujeron los adaptadores ${ }^{13}$.

Tampoco era la primera vez que se utilizaba música en una función de Entre bobos: en 1871, el compositor Cristóbal Oudrid había preparado una música incidental (de la que conservamos una Nueva rondalla aragonesa editada para canto o piano sólo), que podría haber tenido como objeto alguna de las representaciones de la obra.

Sin embargo, la comedia (en la que don Antonio obliga a su hija Isabel a casarse con el viejo y avaro don Lucas para poder saldar sus deudas, sin saber que la joven está ya enamorada de un galán que resulta ser don Pedro, primo pobre de don Lucas, al que éste quiere casar con su hermana, doña Alfonsa), se prestaba al contraste de voces, de solos y conjuntos tanto como The Merry Wives of Windsor de Shakespeare, que había sido ya puesta en música por Otto Nicolai (en alemán) e iba serlo de nuevo, por Verdi (Falstaff) en italiano.

La crítica fue unánime en la aceptación de la obra y en destacar tanto el trabajo de los adaptadores, como el del compositor. Llama la atención que José de Laserna, en El Imparcial, a pesar de felicitar a los autores y repetir (es lugar común en los críticos) la buena idea de adaptar textos del teatro áureo al lírico («la tradicional zarzuela», dice Laserna), se lamente de la elección de Entre bobos, pues ésta es

Una comedia de carácter, aunque de las llamadas «de figurón»; toda la acción gira en derredor de un solo personajes, don Lucas, con cierta monotonía que presta estrecho y reducido campo para el desarrollo de las facultades del compositor. [...] Se ha dicho que donde acaba el poder de expresión de la palabra, comienza el de la música. En obras tales, la palabra llegó al non plus ultra ${ }^{14}$.

J. Arimón, en El Liberal, destacaba que Luceño y Fernández Shaw habían «conservado en su integridad los dos primeros actos, reemplazando el tercero por otro completamente original y más en armonía con la índole del género a que la nueva obra pertenece» ${ }^{15}$. Esta sustitución, piensa el crítico, evita «al músico la terrible competencia que en los actos anteriores le hiciera la maravillosa poesía del gran dramaturgo toledano». Reconoce asimismo que los cambios realizados «ofrecen al compositor ancho campo donde puede lucir las alas de su fantasía creadora». Sobre ello hemos de volver.

Como refundidor, Tomás Luceño se había ocupado de obras de los principales autores del XVII A secreto agravio, secreta venganza y El mayor monstruo, los celos, de Calderón; Donde hay agravios, no hay celos, de Rojas, y El examen de maridos y La hermosa fea, de Lope. El único caso con música es el que nos ocupa ${ }^{16}$. Tal vez ello justificase la colaboración con Fernández Shaw, que no se había ocupado, por su parte, de los

${ }^{12}$ Don Lucas del Cigarral, zarzuela en tres actos y en verso. Las citas, a partir de aquí, se hacen siempre por la edición citada en la bibligrafía.

${ }_{13}$ C. Menéndez Onrubia, 2007a, p. 418. De hecho, es el único título de zarzuela basado en el teatro aurisecular aducido por Espín Templado en su panorama de 1987 (p. 32).

14 J. Laserna, «Los teatros. Parish. Don Lucas del Cigarral.»

15 S. Arimón, «Teatro de Parish. Don Lucas del Cigarral.»

16 J. Vidanes, 2003, pp. 29-37, J. Prieto Marugán, 2012, pp. 232-243. 
autores áureos, pero sí de Ramón de la Cruz (Las castañeras picadas) o de Shakespeare (Las bravias, a partir de The Taming of the Shrew), en ambos casos en género chico y con música de Chapí.

No en balde se anuncia la obra como «refundición», toda vez que Don Lucas mantiene mucho de Entre bobos, sobre todo para pertenecer a otro género, el teatro lírico. A éste responden las escenas iniciales y finales del primer acto, el arranque «costumbrista» del segundo acto (que ayuda, además, a abreviar escenas) y, sobre todo, el tercero.

En la escena inicial, la presencia de unos cómicos que se despiden de la venta permite, además de introducir la escena inicial con coro, aprovechar el aspecto metateatral de la Comedia e introducir el recurso del «teatro dentro del teatro» que va a tener tanta importancia en el desenlace de la zarzuela.

La escena II, tras la salida de los cómicos, ya parece pertenecer a Entre bobos, aunque en realidad no hay versos de Rojas hasta la carta, que originalmente lee don Antonio ya en presencia de Cabellera, y que se adelanta a esta escena, pues es la propia Isabel quien la lee a Andrea. Es preciso destacar, sin embargo, la excelente imitación de estilo que supone.

Mayor va a ser la contribución de Rojas a partir de la escena en la que Cabellera hace un retrato de don Lucas: se mantienen también el diálogo siguiente y la descripción de don Pedro, que se abrevia para incluir un dato de interés: «conciertan su casamiento / con la hermana de don Lucas, / doña Alfonsa de Toledo» (I, III, p. 24).

Interesante es que se retrase la entrada de don Pedro hasta pasada la de don Lucas, lo mismo que el diálogo entre don Luis y Carranza. La entrada del protagonista, convertida en número musical, avanza en el tiempo y cobra mayor importancia. En el aspecto textual, se mantienen los primeros versos, los que se oyen dentro ( «-Ha de la venta!

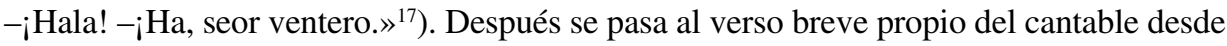
la intervención de don Antonio:

$$
\begin{aligned}
& \text { ¿Lo ves? ¡Lo estaba viendo! } \\
& \text { Su cólera revienta } \\
& \text { y la emprende a estocadas } \\
& \text { con la gentuza necia }{ }^{18} \text {. }
\end{aligned}
$$

La siguiente escena hablada, ya con don Lucas en la venta, tiene versos propios, pero modelados sobre los del original: silva con versos pareados que en Entre bobos comenzaba con el diálogo entre don Luis y Carranza (v. 541) y que en Don Lucas, atrasado dicho diálogo, se restablece tras el número musical, con versos nuevos, pero excelentemente imitados:

PEDRO:

¿Qué ha ocurrido, señor, tan de repente, que indignado venís con tanta gente?

\footnotetext{
17 Acto primero, escena V, p. 25.

18 Acto primero, escena V, p. 26.
} 


\section{VENTERO:}

¿Cuál es la vil canalla

que de respeto y de temor no calla

ante señor tan bravo y tan apuesto?

\section{LUCAS:}

¡Pronto me conociste!

\section{VENTERO:}

Pues, ¿qué es esto?

Salid todos aprisa, con orden y callados, como en misa ${ }^{19}$.

Al final de la escena, se van usando versos de Rojas hasta que, ya en la Escena VII, se vuelve al texto de Entre bobos.

En cuanto al acto segundo, comienza, como he mencionado ya, con una escena de carácter costumbrista entre el Mesonero, su Criado y Espolique, mozo al que el Ventero ha dejado vigilando porque se figura «que hay enredo» (II, I, p. 43). Con esta escena, se suplen unos versos que más adelante dice Cabellera:

No sea que el huésped nos sienta, que es el huésped más cocido que hay en Illescas y siente dentro en su casa un mosquito ${ }^{20}$.

En la escena II se toma la primera de la segunda jornada de Rojas, casi desde la acotación. Sirve de introducción a la romanza del tenor («Era en julio caluroso»), en la que se intercalan partes habladas con versos literales de la comedia, tras uno nuevo que sirve de transición:

Una voz me encamina y más me llama.

Piso por las orillas y, tan quedo que pensé que pisaba con el miedo.

Voy apartando una y otra rama $\mathrm{y}$, en el tibio cristal de la ribera, su deidad contemplé de esta manera: todo el cuerpo en el agua, hermoso y bello, fuera el rostro y en roscas el cabello. Deshonesto, el cristal que la gozaba, de vanidad al Soto la enseñaba, mas si de amante el Soto la quería, por gozársela él toda, la cubría. (II, II, p. 45) 21 .

Así, el que Isabel cuente en el primer acto de Don Lucas cómo la salvó don Pedro, permite aligerar ahora esa parte narrativa, menos adecuada para ser puesta en música, por la mayor concentración y brevedad que requiere el verso para ser cantado. Llama la

\footnotetext{
19 Acto primero, escena VI, p. 29.

20 Acto segundo, vv. 1137-1140.

${ }^{21}$ Corresponden a los vv. 999-1010 de la comedia.
} 
atención la mezcla del recitado en mitad de la romanza (procedimiento muy poco habitual en la zarzuela) con adaptaciones «cantables» de otros versos de Rojas en los que los cambios de ritmo recuerdan a los «experimentos» rosalianos estudiados por Marina Mayoral y que deben tener que ver con la puesta en música ${ }^{22}$.

Acabada la romanza, prosigue el texto de Rojas literalmente hasta unos versos que se insertan para justificar la salida de escena de Cabellera y Andrea, de modo que soprano y tenor queden a solas para su dúo, que también va introducido por versos de Rojas hasta el de doña Isabel «Hablad quedo y ved que estamos...» (v. 1203), que se convierte en el cantable

¡Por Dios, don Pedro,
bajad la voz:
pueden oírnos,
callad, por Dios!

Los versos de Rojas se retoman desde el siguiente («Templar la voz no resisto», v. 1204), como si el dúo completo glosara sólo ese verso de doña Isabel.

En el resto de la escena, se sugiere un corte en los versos de doña Isabel, entre «¿Qué os daña a vos que él me quiera?» y «...y él es el aborrecido?» (vv. 1261-1280). Se abrevia algún verso más y se retoma el texto cuando Andrea (aquí Andrea y Cabellera) avisan de que se abre la puerta del cuarto «de don Luisillo» (v.1306).

De nuevo se abrevia el texto en el diálogo entre don Luis y Carranza, para dar lugar a una serenata de don Luis, convertido en barítono, ante la puerta de doña Alfonsa, que él cree la de Isabel («Oye con el alma mi voz») y prosigue (II, VII, p. 58 y ss.) con la salida de doña Alfonsa (v. 1375).

Un poco más adelante, y siguiendo una tradición de dúo cómico entre tenor y bajo (podrían citarse ejemplos en la tradición operística como los de Die Entführung aus dem Serail de Mozart $o$ el Barbiere rossiniano, pero también el mucho más cercano de $E l$ juramento de Gaztambide) toma este formato la escena en que Cabellera intenta distraer a don Lucas pidiéndole que le lea su comedia («iAfuera, / Cabellera!», II, VIII, p. 61) ${ }^{23}$.

El final del acto, en el que se mantiene también el texto de Rojas, no tiene música: la cantidad de números musicales anteriores lo hace comprensible: el espectáculo podría acercarse a la ópera y eso no era del gusto del público. Volviendo, para variar, a las tradiciones del verso hablado del XVII, todos los personajes cierran diciendo a la vez: «i la venta está el diablo / y entre bobos anda el juego!!».

Como ya he mencionado, el tercer acto incluye novedades que comienzan por el lugar de la acción: si hasta aquí se han mantenido los lugares de Entre bobos, el tercer acto de Don Lucas del Cigarral sustituye el camino del original, con el que Rojas dejaba el futuro incierto (no se ha llegado al término del viaje ni se sabe si el final de la obra supone la felicidad de los enamorados) por el cigarral del protagonista, donde se desarro-

\footnotetext{
${ }^{22}$ Refiriéndose a En las orillas del Sar, escribe la profesora Mayoral: «encontramos [...] ritmos y combinaciones originales, que se desarrollaron después ampliamente en el Modernismo. No creo que existiera en Rosalía un prurito de originalidad en el empleo de formas nuevas, sino, más bien, que su afición y buenas dotes para la música [...] la llevaron a experimentar espontáneamente en su poesía ritmos poco frecuentes.» (1986, p. 39).

${ }^{23}$ Corresponde a los vv. 1509-1540 de Entre bobos.
} 
lla una fiesta de bienvenida para la esposa de don Lucas. Nos encontramos aquí con un cambio en el punto de vista frente a don Lucas, que se convierte en el personaje maduro y sensato que renuncia al amor en favor de la juventud, como don Diego en El sí de las niñas, con la que no en balde se ha relacionado Entre bobos ${ }^{24}$.

Al considerar los cambios realizados en este acto, hay que tener en cuenta que Profeti señala sobre la comedia original que «desde la segunda jornada se trata de 'retrasar' el desenlace final y al mismo tiempo de prepararlo adecuadamente, según una ley fundamental de la comedia» y que «el inicio de la jornada tercera (vv. 1757-2002) está dominado por la parálisis, cuyo eje son los celos sucesivos de los varios componentes de las parejas $»^{25}$. Con un punto de vista similar, los adaptadores han optado por una fiesta y por un cambio de actitud del protagonista: ya en su casa, el carácter de don Lucas se ve repentinamente ennoblecido. Sus palabras a los convidados no dan lugar a duda:

Y voacedes, señores, entretanto

que comienzan las fiestas que previno

el amor que a Isabel rindió mi alma,

recorred con holgura y sin cumplido

los jardines que fueron mis amores,

hoy por los de Isabel sustituidos. ${ }^{26}$

En una escena hablada, que corresponde al arranque de la tercera jornada de Entre bobos, pero de la que apenas sigue el texto, don Lucas explica este cambio, descubriendo a su futuro suegro lo que esconde su rudeza:

Yo soy, como visto habréis,

lo que se llama un hidalgo:

asperote por de fuera,

mas por dentro soy un santo.

$[\ldots]$

Tiénenme por muy roñoso

y es verdad, porque declaro

que, a fe mía, no me gusta

lo que gané con trabajo

tirarlo a tontas y a locas,

como si fuese heredado.

Soy viejo, mal mozo y feo

(en esto la palma gano),

carnes secas, paliducho,

de genio severo y agrio;

mas, como tengo dineros,

todos me encuentran muy guapo. ${ }^{27}$

\footnotetext{
24 «Fue Federido Ruz Morcuende quien aludió a esa comedia [Entre bobos] en 1924, al editar las piezas más importantes de Moratín.» (J. García Barrientos, 2007, p. 151).

25 Profeti, 1998, pp. XLIV y XLV.

26 Acto tercero, escena segunda, p. 79. Se refirió ya a este cambio Ricardo de la Fuente Ballesteros, 1990, p. 211.

27 Acto tercero, escena IV, p. 80.
} 
Creo que tenemos que situar este cambio dentro de la corriente de valoración positiva del figurón que se ha observado en el estudio de comedias del siglo XVIII ${ }^{28}$, desde los figurones de Cañizares (según Arellano y García Ruiz ${ }^{29}$ ), en la que los valores defendidos por el figurón pasan a ser positivos y a constituir una lección para los que se burlaban de él. Es una corriente que, como ya ha señalado Olga Fernández Hernández, llega a las tragedias grotescas de Arniches e incluso al repertorio posterior escrito para cómicos como Enrique Chicote, Paco Martínez Soria, Juanito Navarro o Quique Camoiras hasta ya muy avanzado el siglo XX:

Todavía, fuera incluso del siglo XVIII, podríamos rastrear algunos avatares de los figurones positivos finiseculares: don Frutos de El pelo de la dehesa de Bretón de los Herreros, en el siglo XIX y en el XX, el viejo cateto aragonés de La ciudad no es para mí; incluso, alguna reencarnación del tipo del avaro en El Tío Miserias de Arniches. Pero aunque sobreviva algún eslabón perdido del estereotipo figuronil, lo cierto es que la comedia de figurón como subgénero o especie teatral no superó el XVIII ${ }^{30}$.

Luceño y Fernández Shaw buscan también humanizar al figurón en el sentido señalado por Teresa Julio: a medida que se humanicen, transmitirán su humanidad a quienes le rodean ${ }^{31}$.

De acuerdo con esta tendencia, el «castigo» que don Lucas impone a la pareja enamorada en la comedia de Rojas, permitiéndoles casarse aunque sean pobres, se suaviza con la declaración de perdón y de ayuda que hace a continuación. Comparemos los versos del original y de la refundición:

28 Véase Fernández Hernández, 2008, p. 337 y Angulo Egea, 2008, p. 380.

${ }^{29}$ I. Arellano y V. García Ruiz, «Calderón y la comedia de figurón», en su edición de Guárdate del agua mansa, Kassel, Reichenberger, 1989, p. 42, cit. Por O. Fernández Hernández 2007, p 337.

30 O. Fernández Hernández, 2007, p. 371.

31 En este sentido, el figurón sigue siendo el centro de la acción, que ahora transmite un humanidad primaria, pero cálida como antes contagiaba «su figuronismo», en acertada expresión de M. ${ }^{a}$ Teresa Julio (2007, p. 152). 
ENTRE BOBOS III, vv. 2725-2754

Vos sois, don Pedro, muy pobre, y a no ser porque en mi halláis el arrimo de pariente, perecierais.

\section{DON PEDRO:}

Es verdad.

\section{DON LUCAS:}

Doña Isabel es muy pobre: por ser hermosa no más yo me casaba con ella, pero no tiene un real de dote.

\section{$[\ldots]$}

Pues dalda la mano al punto, que en esto me he de vengar: ella, muy pobre; vos, pobre, no tendréis una hora de paz; el amor se acaba luego, nunca la necesidad; hoy, con el pan de la boda, no buscaréis otro pan; de mí os vengáis esta noche, y mañana, a más tardar, cuando almuercen un requiebro, y en la mesa, en vez de pan, pongan una fe al comer $y$ una constancia al cenar, $\mathrm{y}$, en vez de galas, se ponga un buen amor de Milán, una tela de «mi vida», aforrada en «¿me querrás?», echarán de ver los dos cuál se ha vengado de cuál.
DON LUCAS (III, XI, p. 101)

Dura lición me habéis dado, pero quiérome vengar... ¿Cómo? Dejándooos casar, y así quedo bien vengado.

(A DON ANTONIO y a todos los demás.)

Doña Isabel es divina, don Pedro no tiene un real $\mathrm{y}$ amor sin dinero es mal que no encuentra melecina. (A DON ANTONIO.)

*Ya veréis, suegro... en boceto,

*qué vida van a pasar:

*un «te adoro» de almorzar

*y de comer, un soneto.

* De cenar, señas de amor,

*de vestir, variados trajes...

*de celos, riñas y ultrajes

*que es tela muy inferior.

El amor se irá pasando,

la escasez irá creciendo, se irán luego aborreciendo y así yo me iré vengando.

(Creciendo por instantes su cólera.)

Y cuando vuelvan a mí en demanda de perdón...

(Más enfadado que antes y colocándose entre los dos.)

Juro por mi salvación...

(Transición llena de ternura, y abrazándolos.)

...ique he de deciros que sí!

Porque al pretender tu mano, lo hice amándote con fe $\mathrm{y}$, ya que grande empecé, quiero acabar soberano.

Este cambio del punto de vista sobre don Lucas se produce dentro de un acto tercero casi completamente nuevo. Probablemente fue aquí donde fue mayor el trabajo de Fernández $\mathrm{Shaw}^{32}$.

Volviendo al comienzo del acto, la acotación se refiere a un «jardín frondoso y elegante ${ }^{33}$ que poco o nada tiene que ver con el carácter que se le supone a Don Lucas (el que había en el original, pero también el que describe Cabellera en el primer acto y demuestra el propio personaje en los actos anteriores). Del mismo modo, llama la aten-

\footnotetext{
32 Conservamos de su mano una copia de este acto, Madrid, BNE, MSS 21360 / 1.

33 Acto tercero, p. 74. Todas las citas del arranque del acto corresponden a esta página.
} 
ción que «damas y caballeros, amigos de Don Lucas», se paseen por la escena alabando el lugar:

\author{
Bien puede don Lucas \\ lucir con orgullo, \\ en lugar de su propio apellido, \\ el apellido nuevo y flamante \\ del Cigarral.
}

Cigarral más hermoso que el suyo

no conocí jamás.

Del mismo modo, la acotación de la segunda escena propone la presencia de «Aldeanos y aldeanas» que «dan muestras de su regocijo, tirando aquellos los sombreros al aire y haciendo todos vivas muestras de júbilo y simpatía» ${ }^{34}$.

También es extraño que el coro comente el proyectado matrimonio (que don Lucas ha intentado, en el original y en esta adaptación, mantener en secreto), dado que no se hace por el carácter de don Lucas, sino por la diferencia de edad entre los novios:

$\begin{array}{ll}\text { ELLOS } & \text { Nadie ignora que don Lucas... } \\ \text { ELLAS } & \ldots \text { es un tronco carcomido... } \\ \text { ELLOS } & \ldots \text {.es un árbol ya sin hojas } \\ & \text { y no hay pájaro que quiera } \\ & \text { fabricar en él su nido. } \\ & \text { Nadie ignora que Isabel } \\ \text { ELLAS } & \text { es un rosal siempre en flor } \\ & \text { que prodiga con encanto celestial } \\ & \text { su perfume embriagador. } \\ & \text { con las ramas carcomidas de los árboles ya viejos } \\ & \text { los capullos y las flores del rosal } !^{35}\end{array}$

Del mismo modo, el coro está al tanto del valor de don Pedro que es «un árbol muy florido» y en el que «una tórtola quisiera / fabricar en él su nido». ${ }^{36}$

Finalmente, el coro se remata con una referencia costumbrista, muy en la tradición de la zarzuela, que se imbrica, además, en la del romanticismo poético español a través de la célebre leyenda A buen juez, mejor testigo, de Zorrilla: «iSanto Cristo de la Vega! $/$ ¡De tal trance / libera nos!» ${ }^{37}$.

\footnotetext{
${ }^{34}$ Acto tercero, escena segunda, p. 77.

35 Acto tercero, escena primera, p. 75.

36 Acto tercero, escena primera, p. 75.

37 Acto tercero, escena primera, p. 75.
} 
Más allá de los cantables, el acto entero demuestra la presencia de otra mano, pues no se sigue tan de cerca el verso de Rojas, sino que se adapta de forma muy libre:

ENTRE BOBOS (III, vv. 1841-1864)

Lo primero: envié a decir que saliese con cuidado de Madrid y se pusiese una máscara al recato, y ella se puso por una media mascarilla, tanto que se le vio media cara, desde la nariz abajo; lo segundo, os supliqué que no vinierais, enviando, de que a Isabel admitía, un recibo ante escribano, $\mathrm{y}$ os venistis, no sabiendo que yo he de vestirme llano, pues la tela de mujer no ha menester suegro al canto; lo tercero, luego al punto que me vio, se fue de labios y me dijo mil requiebros por mil rodeos extraños, y una mujer, cuando es propia, ha de andar camino llano, que no ha de ser hablador el amor que ha de ser casto; $[\ldots]$
DON LUCAS (III, IV, p. 81)

Primeramente, ¿por qué don Luis, doquiera que vamos sigue a Isabel y no cesa de dirigirle vocablos de amor, que ella escucha siempre sin enojo y sin enfado? Segundamente, ¿por qué, la primera vez que hablamos en la venta, contestome con amoroso descaro, cuando el rubor es más propio del amor, si ha de ser casto? Terceramente, ¿por qué ese mozuelo fue osado de quererla hablar anoche dentro de su mesmo cuarto?

Diferente análisis de la situación para preparar un final con diferentes consecuencias.

Similar es lo que ocurre con el entremés que se representa en la obra, que se dice obra de Quiñones («porque pedí a Benavente, / el poeta celebrado») ${ }^{38}$, pero para el que no encuentro equivalente en las obras del ilustre entremesista. Sí hallo, en cambio, un cierto recuerdo del Viejo celoso cervantino en la representación, urdida para que don Lucas entienda que no es conveniente a su edad casar con mujer joven. El entremés debe de ser un pastiche realizado Fernández Shaw: el estilo recuerda más al verso cómico del XIX que al del XVII:

\section{AURORA:}

Digo que aunque me mates le desprecio, por avaro, rüin, caduco y necio.

\section{ROQUE:}

No pido que le quieras, lo que pido es que finjas amor muy bien fingido: que le hagas carantoñas, que le hables

\footnotetext{
${ }^{38}$ Acto tercero, escena V, p. 84.
} 
en concetos tiernísimos y amables, que le beses su frente despejada...

AURORA:

¿Despejada, señor?... ¡No dices nada!

El viejo que se case con doncella, tendrá la frente como quiera ella ${ }^{39}$.

Como se puede observar por el ejemplo, los autores, en contraste con la cuidadosa imitación de versos de Rojas en los actos anteriores, han optado en el entremés por imitar la situación sin hacerlo con el verso.

Hasta aquí un repaso inicial a esa adaptación de Entre bobos anda el juego, texto que espero editar próximamente.

\section{BiBLIOGRAFÍA}

Álvarez Barrientos, J., 2007, «Tras ser desfigurado, Francisco de Rojas Zorrilla entre en el Parnaso», Revista de Literatura, LXIX, 137, pp. 141-162.

Angulo Egea, María, 2008. «Los figurones de Luis Moncín: tradición y modernidad» en Luciano García Lorenzo (ed.), El figurón, Madrid, Fundamentos / RESAD, pp. 373-401.

Arimón, J., «Teatro de Parish. Don Lucas del Cigarral.» en El Liberal, 19-II-1899, p. 3.

Cotarelo y Mori, Emilio, 1934. Historia de la zarzuela o sea, el drama lírico en España, desde su origen a fines del siglo XIX, Madrid, Tipografía de Archivos.

Espín Templado, M. ${ }^{a}$ Pilar, 1987. «La zarzuela: esquema de un género español», Andrés Amorós (ed.), La Zarzuela de cerca, Madrid, Espasa-Calpe, Austral, pp. 21-35.

- 1993. "Zorrilla: del teatro declamado a la zarzuela», Javier Blasco, Ricardo de la Fuente y Alfredo Mateos (eds.), José Zorrilla, una nueva lectura, Valladolid, Fundación Jorge Guillén / Universidad de Valladolid, pp. 299-305.

— 2009. «Los clásicos y la zarzuela: de refundiciones, adaptaciones y parodias», Joaquín Álvarez Barrientos, Óscar Cornago Bernal, Abraham Madroñal Durán y Carmen MenéndezOnrubia (eds.), En buena compañía. Estudios en honor de Luciano García Lorenzo, Madrid, Consejo Superior de Investigaciones Científicas, pp. 1095-1111.

FERnÁndeZ HernándeZ, Olga, 2007. «Pervivencia y evolución de tipos y formas: de los figurones 'especiales' de Cañizares al figurón 'inexistente' de Iriarte» en Luciano García Lorenzo (ed.), El figurón, Madrid, Fundamentos / RESAD, pp. 335-371.

Fernández SAN Emeterio, Gerardo, 2012. «Elementos ideológicos, estéticos y literarios en Gloria y peluca, de Villa del Valle y Barbieri», Dicenda, 30, 115-127.

Fuente Ballesteros, Ricardo de la, 1990. «La pervivencia de la comedia áurea en la zarzuela», Cuadernos de Teatro Clásico, 5, pp. 209-217.

García Lorenzo, Luciano, 2007. «De la fortuna escénica de Rojas Zorrilla en los teatros de Madrid», Revista de Literatura, 137, pp. 235-247.

Julio, M. ${ }^{a}$ Teresa, 2008. «Ridendo castigat mores: figurón y figuras en Entre bobos anda el juego», en Luciano García Lorenzo (ed.), El figurón, Madrid, Fundamentos / RESAD, pp. 149-163.

Laserna, José. «Los teatros. Parish. Don Lucas del Cigarral» en El Imparcial, 19-II-1899, p. 3.

Luceño, Tomás y Carlos Fernández Shaw. Don Lucas del Cigarral, zarzuela en tres actos y en

39 Acto tercero, escena XI, Entremés, p. 94. 
verso, refundición de la comedia de D. Francisco de Rojas «Entre bobos anda el juego», Madrid, Sociedad de Autores, 1899.

Mayoral, Marina (1986). Introducción a Rosalía de Castro, En las orillas del Sar, Madrid, Castalia, Clásicos Castalia, 90.

MenÉndez OnRubia, Carmen, 2007a. «El figurón en escena: Donato Jiménez» en Luciano García Lorenzo (ed.), El figurón, Madrid, Fundamentos / RESAD, pp. 405-420.

- 2007b. «Memoria de actores: Entre bobos anda el juego en la escena del Teatro Español de Madrid (1895-1896)», Revista de Literatura 137, pp. 219-234.

Peña, Aniano, 1986. Introducción a José Zorrilla, Don Juan Tenorio, Madrid, Cátedra, Letras Hispánicas.

Prieto Marugán, José, 2012. El teatro lírico de Carlos Fernández Shaw, Madrid, Ediciones del Orto.

Profeti, M. a Grazia, 1998. «Estudio preliminar» y «Prólogo» a Francisco de Rojas Zorrilla, Entre bobos anda el juego, Barcelona, Crítica, pp. IX-LIX.

Vidanes, Julio, 2003. Tomás Luceño, teatro breve. Estudio y erudición, tesis doctoral, Madrid, Universidad Complutense. 